\title{
Density Evolution for Multistage CDMA Multiuser Detector
}

\author{
Toshiyuki Tanaka \\ Department of Electronics \& Information Engineering, Tokyo Metropolitan University \\ 1-1 Minami-Osawa, Hachioji, Tokyo 192-0397, Japan; and \\ Neural Computing Research Group, Aston University, Aston Triangle, Birmingham, B4 7ET United Kingdom. \\ e-mail: tanaka@eei.metro-u.ac.jp
}

\section{INTRODUCTION}

An analytical framework to analyze the stage-by-stage detection dynamics of the multistage CDMA multiuser detector [1] is presented. The density evolution idea is applied to analyze the multistage detector. Message distribution is treated basically by Gaussian approximation, but interstage correlation of messages is systematically taken into account, which turns out to provide significant improvement.

\section{Multistage Detector}

We consider the following basic fully-synchronous $K$-user baseband CDMA channel model with perfect power control,

$$
r^{\mu}=N^{-1 / 2} \sum_{k=1}^{K} s_{k}^{\mu} x_{k}+\sigma_{0} n^{\mu} \quad(\mu=1, \ldots, N)
$$

where $r^{\mu}$ is the received signal at chip interval $\mu$, and $x_{k} \in$ $\{-1,1\}$ and $\left\{s_{k}^{\mu} ; \mu=1, \ldots, N\right\}$ are the information bit and the signature sequence of user $k(k=1, \ldots, K)$, respectively. $\sigma_{0}^{2}$ is the variance of channel noise, and $n^{\mu} \sim N(0,1)$. The multistage CDMA multiuser detector [1] updates the tentative decision $\hat{x}_{k}^{(t)}$ at stage $t$ by

$$
\hat{x}_{k}^{(t)}:=\operatorname{sign}\left[h_{k}-\sum_{k^{\prime} \neq k} W_{k k^{\prime}} \hat{x}_{k^{\prime}}^{(t-1)}\right]
$$

where $h_{k} \equiv N^{-1 / 2} \sum_{\mu=1}^{N} s_{k}^{\mu} r^{\mu}$ is the matched filter output for user $k$, and $W_{k k^{\prime}} \equiv N^{-1} \sum_{\mu=1}^{N} s_{k}^{\mu} s_{k^{\prime}}^{\mu}$ is the correlation of the signature sequences of users $k$ and $k^{\prime}$.

\section{Analysis and Results}

We have shown that the multistage detector can be regarded as an approximate sum-product algorithm, in which $\hat{x}_{k}^{(t)}$ and $u_{k}^{(t)} \equiv h_{k}-\sum_{k^{\prime} \neq k} W_{k k^{\prime}} \hat{x}_{k^{\prime}}^{(t)}$ serve as messages between variable and chip nodes. This observation suggests application of the density evolution idea to describe stage-by-stage dynamics of the multistage detector under random spreading assumption and in the large-system limit $(K, N \rightarrow \infty$ while $\beta=K / N$ kept finite). We assume $x_{k}=1$ without loss of generality. Distribution of $\hat{x}_{k}^{(t)}$ is characterized by the overlap $M_{t} \equiv K^{-1} \sum_{k=1}^{K} \hat{x}_{k}^{(t)}$, which is also related to the bit error rate $P_{b}^{(t)}=\left(1-M_{t}\right) / 2$. Distribution of $u_{k}^{(t)}$ is, however, diffcult to characterize, because $u_{k}^{(t)}$ contains the following term which has complex interstage dependence.

$$
N_{k}^{(t)} \equiv \sum_{k^{\prime} \neq k} W_{k k^{\prime}} \hat{x}_{k^{\prime}}^{(t)}=N^{-1} \sum_{\mu=1}^{N} \sum_{k^{\prime} \neq k} s_{k}^{\mu} s_{k^{\prime}}^{\mu} \hat{x}_{k^{\prime}}^{(t)},
$$

Motivated by an analysis on similar systems in neural network literature [2], we decompose $u_{k}^{(t)}$ as $u_{k}^{(t)}=1+N_{0 k}-N_{k}^{(t)}$, and assume the distribution of $N_{0 k}$ and $N_{k}^{(t)}$ to be joint Gaussian,

$$
\left(\begin{array}{c}
N_{0 k} \\
N_{k}^{(t)}
\end{array}\right) \sim N\left(\left(\begin{array}{c}
0 \\
U_{t}
\end{array}\right),\left(\begin{array}{cc}
\sigma_{0}^{2}+\beta & C_{t} \\
C_{t} & S_{t}^{2}
\end{array}\right)\right)
$$

and derive time evolution equations for the parameters by taking into account correlations between terms at adjacent stages only. The resulting equations are

$$
\begin{aligned}
& M_{t+1}=\sum_{x= \pm 1} \frac{1+x M_{t-1}}{2} F\left(\frac{1-U_{t}(1-x)}{V_{t}}\right) \\
& U_{t+1}=\frac{\beta}{V_{t}} \sum_{x= \pm 1} \frac{1+x M_{t-1}}{2} F^{\prime}\left(\frac{1-U_{t}(1-x)}{V_{t}}\right) \\
& V_{t}^{2}=\sigma_{0}^{2}+\beta-2 C_{t}+S_{t}^{2}, \quad C_{t}=\beta M_{t}+\left(\sigma_{0}^{2}+\beta-C_{t-1}\right) U_{t} \\
& S_{t}^{2}=\beta+V_{t-1}^{2} U_{t}^{2}+2 \beta M_{t}\left(1-M_{t-1}\right) U_{t}
\end{aligned}
$$

where $F(z) \equiv \sqrt{2 / \pi} \int_{0}^{z} e^{-u^{2} / 2} d u$. Figure 1 shows that the computer simulation results $(\beta=0.5)$ are approximately reproduced by the density evolution. It also shows that the accuracy has been significantly improved by the consideration of the interstage correlations. It should be noted that, even though the Gaussian approximation is itself incorrect, the analytic framework allows us to systematically improve the approximation by taking into account correlations between nonadjacent stages [3].

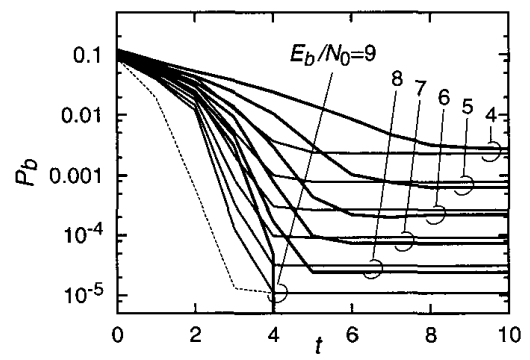

Figure 1: Detection dynamics of multistage detector $(\beta=0.5$; thin lines: density evolution; thick lines: simulations $(K=4000)$; dashed line: density evolution without correlation (shown for $E_{b} / N_{0}=9$ only)).

\section{ACKNOWLEDGMENTS}

I thank Dr. Masato Okada of Brain Science Institute, Riken, Japan, for his helpful discussion. Support from EPSRC research grant GR/N00562 is acknowledged.

\section{REFERENCES}

[1] M. K. Varanasi and B. Aazhang, IEEE Trans. Commun., vol. 38, pp. 509-519, 1990; ibid., vol. 39, pp. 725-736, 1991.

[2] S. Amari and K. Maginu, Neural Netw., vol. 1, pp. 63-73, 1988.

[3] M. Okada, Neural Netw., vol. 8, pp. 833-838, 1995. 